\title{
Solar cycle evolution of ULF wave power in solar wind and on ground
}

\author{
Reko Hynönen $^{1, *}$, Eija I. Tanskanen ${ }^{2,1}$, and Patrizia Francia ${ }^{3}$ \\ ${ }^{1}$ Department of Electronics and Nanoengineering, Aalto University of Technology, 00076 Aalto, Finland \\ 2 Sodankylä Geophysical Observatory, University of Oulu, 99600 Sodankylä, Finland \\ 3 Dipartimento di Scienze Fisiche e Chimiche, Università dell'Aquila, Via Vetoio, Coppito, 67100 L'Aquila, Italy
}

Received 8 April 2019 / Accepted 17 August 2020

\begin{abstract}
The solar cycle evolution of the ultra-low frequency (ULF) power was studied in solar wind and on ground. We aim finding out how the ULF power in interplanetary and on ground magnetic field evolves over the solar cycle 23 (SC23) and how well do they follow each other in monthly time scales. The hourly power of the ULF waves was computed in the Pc5 frequency range $2-7 \mathrm{mHz}$ for years $1998-2008$. The highest wave power in SC23 is found to occur in late 2003 and the lowest at the solar minimum. Ground ULF power follows the IMF power and solar wind speed, particularly well during declining phase. The ULF power in winter exceeds the ULF power in other seasons during the declining phase of SC23, while equinoxes dominate in the ascending phase and the solar maximum. The ground ULF power was found to rise with magnetic latitude from $54^{\circ}$ to $73^{\circ}$, after which Pc5 power decreases towards the polar cap. The Pc5 power in the auroral zone is larger in the nightside than the dayside due to substorm activity implying that magnetotail processes are an important contributor to the nightside ULF power.
\end{abstract}

Keywords: ULF waves / Solar cycle / Space climate / Geomagnetic pulsations / Pc5 waves

\section{Introduction}

Magnetospheric ultra-low frequency (ULF) waves are slow fluctuations of magnetic field within the Earth's magnetospheric cavity, detected on the ground as geomagnetic pulsations. The frequency range of magnetospheric ULF waves is $1 \mathrm{mHz}-$ $5 \mathrm{~Hz}$. The waves can be divided into irregular pulsations (Pi) and continuous pulsations (Pc). This paper focuses on the geomagnetic Pc5 pulsations, defined historically in the range 2-7 mHz (Jacobs et al., 1964).

ULF waves are an important element of the space weather, known to affect magnetospheric and heliospheric processes: they can accelerate, diffuse, and redistribute particles in the magnetosphere and in solar wind (e.g. Takahashi et al., 2012; Menk \& Waters, 2013 and the references therein). ULF waves are thought to be the source of "killer" electrons (higher than $2 \mathrm{MeV}$ ) in the magnetosphere (e.g. Potapov, 2013) and can be used to track plasma mass density of the field line (Clilverd et al., 2003; Menk \& Waters, 2013).

Long-period ULF waves were reported the first time by Anders Celcius already in 1741 (Kangas et al., 1998, but the

\footnotetext{
*Corresponding author: reko. hynonen@aalto.fi
}

first scientific observations of ULF pulsations were reported to occur on ground during the Carrington event of 1859, at Kew Observatory in London (51.5 geog. lat.) (Stewart, 1861). Nowadays, magnetic observations are continuously recorded at magnetic observatories around the world as well as in space.

ULF pulsations are known to vary temporally in time scales from hours and days to years and solar cycles (SC). Different types of pulsations (e.g. Pi and Pc) display different solar cycle variation. According to Fort Churchill data (geom. lat. $68.8^{\circ} \mathrm{N}$, geom. lon. $322.5^{\circ} \mathrm{E}, L \approx 8$ ) the largest Pc5 activity during the SC19 occurred during the sunspot minimum phase (Rao \& Gupta, 1978). Respectively at slightly higher frequencies, Pi2 ( $T=40-150 \mathrm{~s})$ activity has a nearly inverse relationship with sunspot activity over SC18 and SC19 (Saito \& Matsushita, 1968).

Latitudinal and $L$-shell variation of Pc5 pulsations can be addressed with a dense magnetometer network covering a wide latitudinal range from sub-oval to oval and polar cap latitudes. Ground observations (Mathie \& Mann, 2001; Lepidi \& Francia, 2002; Rae et al., 2012; Takahashi et al., 2012) ground show that in mid to high latitudes ( $L$-shells $\sim 1-8$ ) Pc5 pulsations decrease in frequency and increase in power towards higher magnetic latitudes, while at higher than $L=8$ the power 
decreases according to both ground (Yagova et al., 2002) and space observations (Sarris \& Li, 2016).

Preliminary analysis of correlation coefficients across the solar cycle indicated that ULF fluctuations of IMF Bz and solar wind speed should be considered throughout the solar cycle, having the highest correlation with the ground ULF power, 0.83 and 0.80 respectively. However, it is highly likely that these are strongly interdependent. This makes them very difficult to analyse, a problem not resolved in existing literature. We do not consider causality in this study, but show both parameters throughout the solar cycle compared to ground ULF power. The literature outlined below further justifies our choice, as physical mechanisms for driving ground ULF waves and for interdependence with other solar wind parameters have been proposed for each of our two chosen parameters.

Pc5 pulsations are known to correlate well with solar wind speed (Engebretson et al., 1998; Kessel et al., 2004; Pahud et al., 2009, e.g.). Solar wind speed has been shown to have the largest effect to the ground ULF power, the second most important factor being $B_{z}<0$, and the third most one being perturbations of the solar wind density (Bentley et al., 2018). The role of the solar wind ULF waves to the ground Pc5 power has been left unsolved in previous studies, in particular during different solar cycle phases. Theoretically, transverse Alfvénic waves may directly penetrate through the magnetopause (Lee, 1982) transmission, and in certain conditions discrete MHD oscillations in the solar wind have been observed to excite field-line resonances (Stephenson \& Walker, 2002).

Statistical studies have been performed to determine the most important driving quantities in solar wind for ground ULF power. Simms et al. (2006, 2010) used multiple regression analysis on solar wind quantities, finding that the primary driving quantity was the solar wind speed, then secondarily density fluctuations and magnetic field fluctuations. Bentley et al. (2018) studied ground ULF power with solar wind quantities considering their interdependencies, also finding solar wind speed the most important factor, then density fluctuations and southward IMF. However, they could not rule out the effect of IMF fluctuations completely. To explore this further, we investigate the correspondence between the ground ULF fluctuations and the ULF fluctuations within the IMF considering Pc5 frequencies over the SC23 from 1998 to 2008 in monthly time scales. Particularly, we consider the fluctuations of the IMF north-south component because this component, particularly the southward portion, is greatly geoeffective (e.g. Fairfield \& Cahill Jr., 1966; Akasofu, 1980; Simms et al., 2006; Bentley et al., 2018).

Geomagnetic observations used cover geomagnetic latitudes from $53^{\circ}$ to $75^{\circ}$ in the northern hemisphere. The observations from International Monitor for Auroral Geomagnetic Effects (IMAGE) network cover sub-oval, auroral oval and polar cap latitudes. First, we compute the solar cycle variation of ULF power at the ground station of Kevo $(\mathrm{KEV}, L$-shell $\approx 6.2$ ), which is located in the auroral oval latitudes during disturbed geomagnetic conditions. The ULF power in Kevo is compared to solar wind speed and ULF power in the interplanetary magnetic field (IMF). Second, we explore the annual variability of ULF power in IMF and on ground, and study it separately for different solar cycle phases. We conclude this paper by examining the latitudinal variability of the Pc5 power over SC23.

\section{Data and analysis methods}

\subsection{Data}

We have computed ULF wave power (years 1998-2008) in solar wind based on the measurements of IMF, and on ground based on the magnetic field horizontal component $\left(B_{H}\right)$. We used IMF and plasma data in the front of the magnetosphere at L1. The level 2 data from Magnetic Fields Experiment (MAG) (Smith et al., 1998) on-board ACE spacecraft (Chiu et al., 1998; Stone et al., 1998) was used in 1-second resolution. Similarly provided by CDAWeb, solar wind speed Level 2 data at 64-second resolution came from the Solar Wind Electron, Proton, and Alpha Monitor (SWEPAM) (McComas et al., 1998) on-board ACE. All computations regarding the solar wind and IMF were performed in GSM coordinates of ACE at L1.

Magnetic field data of 30 ground magnetic stations from IMAGE network (Tanskanen, 2009) comprehensive were used in ground ULF analysis. The magnetic latitudes, the computed L-shells, and the data coverages of the stations are presented in Table 1. IMAGE network consists of flux-gate magnetometers that measure the ground magnetic field at $1-10 \mathrm{~s}$ time intervals and $0.1 \mathrm{nT}$ resolution in geographic $X Y Z$ coordinates. To convert to the magnetic horizontal component $B_{H}$ we used the formula $B_{H}^{2}=B_{X}^{2}+B_{Y}^{2}$.

For the geomagnetic coordinates of the stations we opted to use the corrected geomagnetic coordinates calculated for the year 2001 (retrieved from http://space.fmi.fi/image/beta/?page= stations), as an approximation of the magnetic latitude for all the years comprised in the study. The corresponding $L$-shell for each station was computed from the magnetic latitude $\theta$ by the formula $L=1 / \cos ^{2} \theta$. The southernmost station, Tarto (TAR, $54^{\circ}$ magn. lat.) locates at $L=3.0$ and the northernmost station, Ny Ålesund (NAL, $75.25^{\circ}$ magn. lat.), at $L=15.4$, although the latter is above the applicable range of the L-shell model $(L=3-10)$.

The solar wind, IMF, and ground magnetic field data of years 1998-2008 cover almost entire solar cycle 23: ascending phase (March 1997 - July 1999), solar maximum (July 1999 September 2002), declining phase (September 2002 - November 2006) and solar minimum (November 2006 - February 2009). The phases of the sunspot cycle were determined by the method developed by Hynönen (2013), which was based on the international sunspot number 1.0 before it was revised and called version 2.0 by Clette et al. (2014). The data were obtained from Solar Influences Data Analysis Center (SIDC), WDC-SILSO, Royal Observatory of Belgium, Brussels. The method gives results that match with the standard way of defining solar cycle minimum and maximum, and gives similar results for the international sunspot number 2.0. Our method is able to define the beginning and end of the ascending and declining phases as well, which is not done by other methods.

\subsection{Sampling and data processing}

For the Fourier analysis, the IMF and ground magnetic field data were initially sampled using 1-hr long samples overlapping every $15 \mathrm{~min}$ for all times between 1998 and 2008, starting at 1 Jan 1998 UT 00:00 - 01:00, 1 Jan 1998 UT 00:15 - 01:15 and continuing so on until 31 Dec 2008 UT 23:00 - 00:00. In the final figures only hourly non-overlapping samples were 
Table 1. Magnetometer data from IMAGE network that is used for computing ground Pc5 pulsation power.

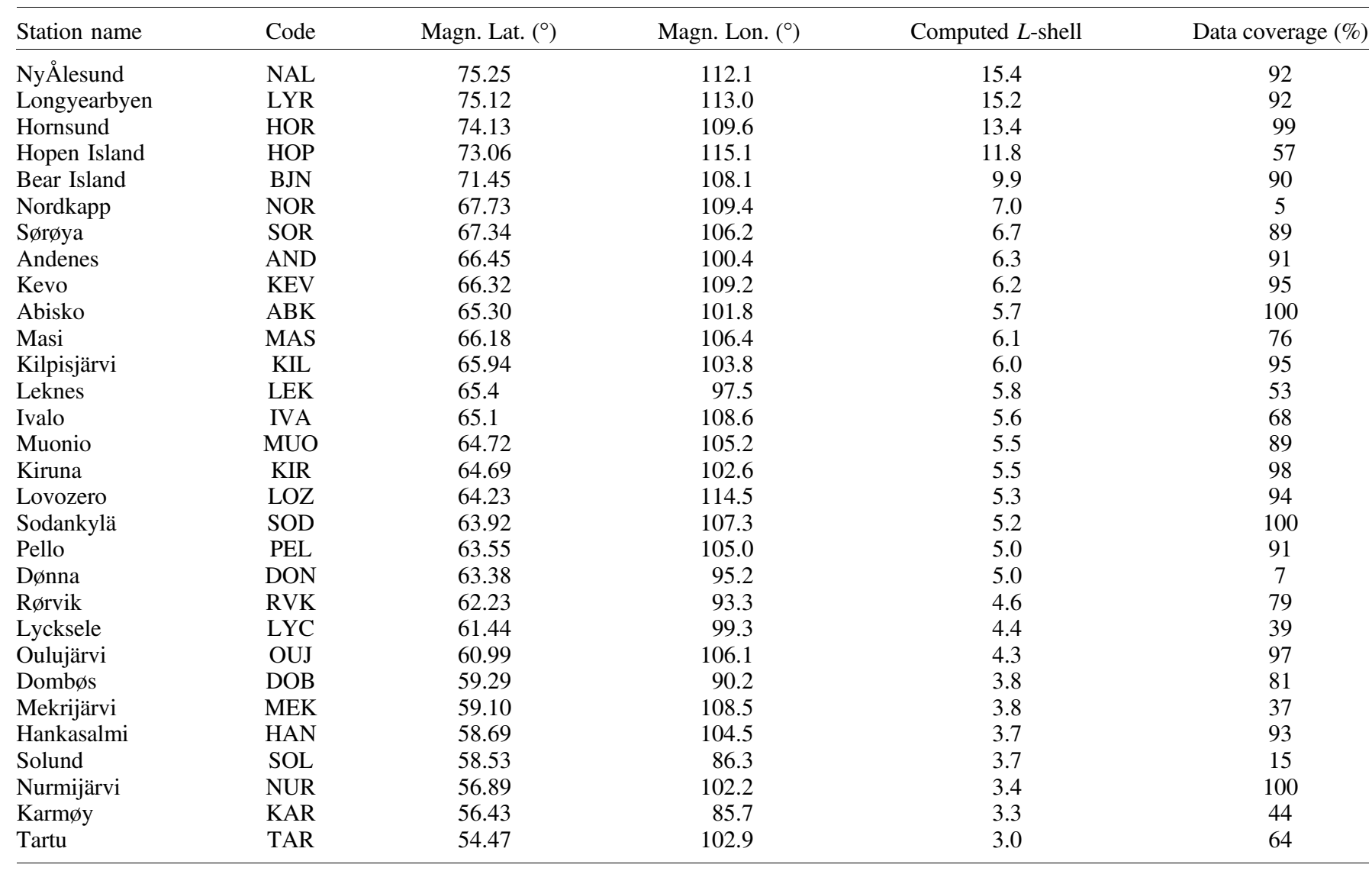

used, because the difference to the result calculated from the overlapping data was very small. Data samples were 360 data points long for ground data, 3600 points for IMF data.

The data samples were probed for missing data, and missing values were filled with linear interpolation using the closest data points on both sides. Samples with more than $10 \%$ missing values were discarded. In total, $0.1 \%$ (about 4 days) of the solar wind magnetic field data was discarded, and $4.7 \%$ (about 189 days) of the ground magnetic data of Kevo respectively, $72 \%$ of the latter in July. Scientists at IMAGE collaborator institutes have checked their magnetic data both automatically and manually for spikes and other errors. In contrast, every sample of ACE spacecraft data was checked and the Augmented Dickey-Fuller test (Greene, 1997) for stationarity was used to make sure that Fourier analysis may be performed validly. We only accepted data samples that passed this test with a $p<0.001$. After discarding data samples with too many missing values and samples failing the stationarity test at $p<0.001$ level, in total $84.2 \%$ of the interplanetary magnetic field data samples were accepted for the analysis. Since no further identification of the types of ULF events is performed, the acquired data samples contain both coherent and incoherent wave packets and may have multiple sources in the solar wind.

\subsection{Fourier analysis}

Power spectra were determined separately for each data sample from ground and space by using Discrete Fourier
Transform (DFT), calculated by Fast Fourier Transform (FFT). FFT was implemented using FFTW library (Frigo \& Johnson, 1998). In this library, DFT is defined with

$$
Y(k)=\sum_{j=1}^{n} X(j) W_{n}^{(j-1)(k-1)},
$$

where $W_{n}=\exp (-2 \pi i / n)$ is one of the $n$ roots of unity.

Before FFT, the mean and linear trend were removed from each data sample to reduce aliasing caused by the initial data sampling by the instrument. Then the end of each sample was padded with zeros until the next power of 2 of the sample length: until 512 points for IMAGE data and 4096 for IMF. Padding to the next power of 2 allows FFT to run at the maximum speed, while slightly increasing resolution in the frequency-domain.

FFT with a Blackman-Harris window (Harris, 1978) was imposed on the prepared data. Power spectra were normalized by the formula $\mathrm{FFT}_{\text {norm }}=\mathrm{FFT} \times \delta t / \operatorname{norm}(w)$, where $\delta t$ is the time resolution of the data $(10 \mathrm{~s}$ for IMAGE/ground data, $1 \mathrm{~s}$ for ACE/spacecraft data) and $w$ is the Blackman-Harris window appropriate for the sample. Then the power spectrum was acquired through squaring (see e.g. Hamming, 1973, Chapter 31). Since the data is real-valued, we used the right-sided spectrum and simply doubled each squared power above frequency 0 to acquire the final power spectrum.

The integrated power of the spectrum was calculated from the power spectra over Pc5 range, $2-7 \mathrm{mHz}$, by summing the 


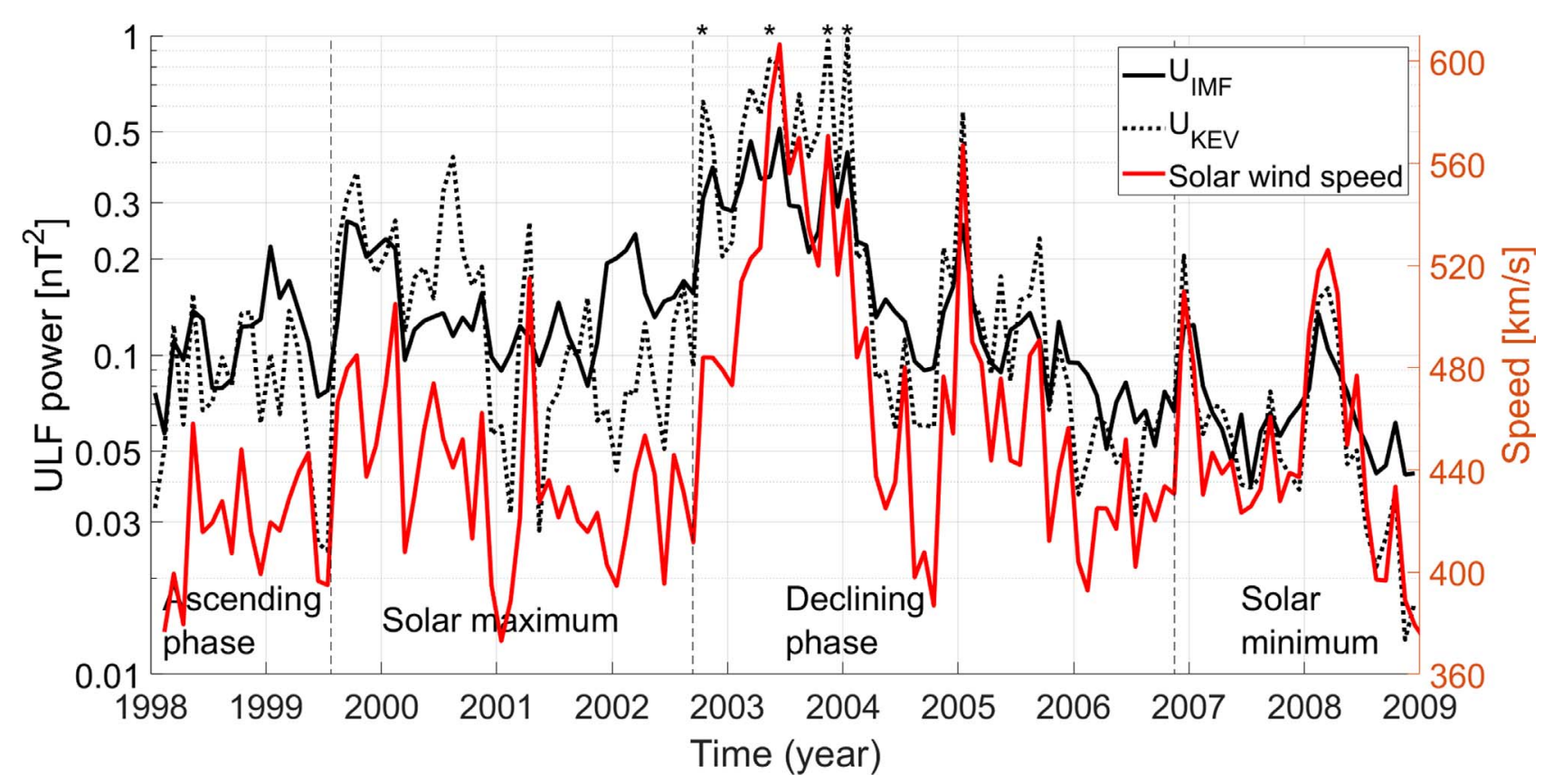

Fig. 1. On the left axis: mean monthly ULF power in the frequency range $2-7 \mathrm{mHz}$ in IMF $B_{Z}$; mean monthly ULF power on the ground at $\mathrm{KEV}$ in the magnetic field component $B_{H}$, with KEV power down-scaled by a factor of 250 . On the right axis: mean monthly solar wind speed. Asterisks imply the four maximum times on the ground. The correlation between IMF and ground ULF wave power is 0.83 for the entire time span studied, 0.31 for the solar maximum, and 0.92 from that onward, i.e. for the declining phase and solar minimum.

squared powers within the frequencies $1.95-7.05 \mathrm{mHz}$ separately for each power spectrum. Since FFTW provides unnormalized DFT, to further normalize the resulting sum it was divided by the zero-padded length of the sample (namely 512 or 4096 for IMAGE ground data and IMF, respectively). The logarithmic integrated power was averaged over each month of years 1998-2008 to acquire the monthly ULF power. These data were used to determine the variability of the ultra-low frequency wave power by the solar cycle phase, magnetic local time, and magnetic latitude. It is essential to realize that the method loses the information about whether the wave power occurs at discrete frequencies or at broad bandwidth. The results of this analysis are presented in the following sections.

\section{Solar cycle and monthly variation of ULF wave power}

The monthly ULF wave power in the total horizontal component $B_{H}$ at Kevo, $U_{\mathrm{KEV}}$, and in the solar wind magnetic field $Z$-component, $U_{\mathrm{IMF}}$, are shown in Figure 1 during the solar cycle 23 (1998-2008).

The largest and the second largest ULF wave power peaks on the ground ( $U_{\mathrm{KEV}}$ in Fig. 1, dashed line) are found in January $2004\left(245 \mathrm{nT}^{2}\right)$ and November $2003\left(241 \mathrm{nT}^{2}\right)$, and the largest and the second largest solar wind magnetic field $Z$-component power $\left(U_{\mathrm{IMF}}\right.$ in Fig. 1, solid line) in June $2003\left(1.03 \mathrm{nT}^{2}\right)$ and March $2003\left(0.94 \mathrm{nT}^{2}\right)$. ULF wave power in Kevo, $U_{\mathrm{KEV}}$, often exceeds $150 \mathrm{nT}^{2}$ between September 2002 and January 2004. This period has four large ULF activity intervals: October $2002\left(156 \mathrm{nT}^{2}\right)$, May $2003\left(211 \mathrm{nT}^{2}\right)$ and two very close together with first in November $2003\left(241 \mathrm{nT}^{2}\right)$ and second in January $2004\left(245 \mathrm{nT}^{2}\right)$. All of them coincide with peaks in solar wind ULF power as well as solar wind speed, and are marked with asterisks in Figure 1. We also find a large peak in the middle of the declining phase in January $2005\left(144 \mathrm{nT}^{2}\right)$.

The largest ULF wave power in the solar wind north-south magnetic field component $B_{Z}$ (Fig. 1, solid line), $U_{\mathrm{IMF}}$, is observed in the beginning of the declining phase of SC23 in 2002-2004 showing the largest values in 2003. This period contains five major power peaks. First ULF power enhancement is in November $2002\left(0.39 \mathrm{nT}^{2}\right)$, month after a minor peak on the ground (October 2002, $156 \mathrm{nT}^{2}$ ). Two double-peaked maxima are seen during 2003, first one in March $\left(0.47 \mathrm{nT}^{2}\right)$ and June $\left(0.51 \mathrm{nT}^{2}\right)$ and second one in November $2003\left(0.44 \mathrm{nT}^{2}\right)$ and January $2004\left(0.83 \mathrm{nT}^{2}\right)$. Both double-peaked maxima have a less intense period in between them, and the peaks are separated by a larger and longer decrease of ULF power in the summerfall $2003\left(0.21 \mathrm{nT}^{2}\right.$ in September 2003). We find smaller but significant ULF power enhancements also in the late declining phase in January $2005\left(0.26 \mathrm{nT}^{2}\right)$, two in the ascending phase in January $\left(0.22 \mathrm{nT}^{2}\right)$ and September $1999\left(0.25 \mathrm{nT}^{2}\right)$ and several annual, almost periodic minor peaks in the late declining phase and the minimum phase from 2005 onward.

The four most clear ULF enhancements on the ground after the mid-2004 occur in January 2005, September 2005, December 2006 and March 2008, marked with asterisks in Figure 1. The power for those ground enhancements were $144,58,51$ and $41 \mathrm{nT}^{2}$ while the power in IMF for the same power enhancements were $0.26,0.11,0.12$ and $0.10 \mathrm{nT}^{2}$, respectively.

Comparatively large ULF powers are detected also during solar maximum and ascending solar cycle phase, but they tend 

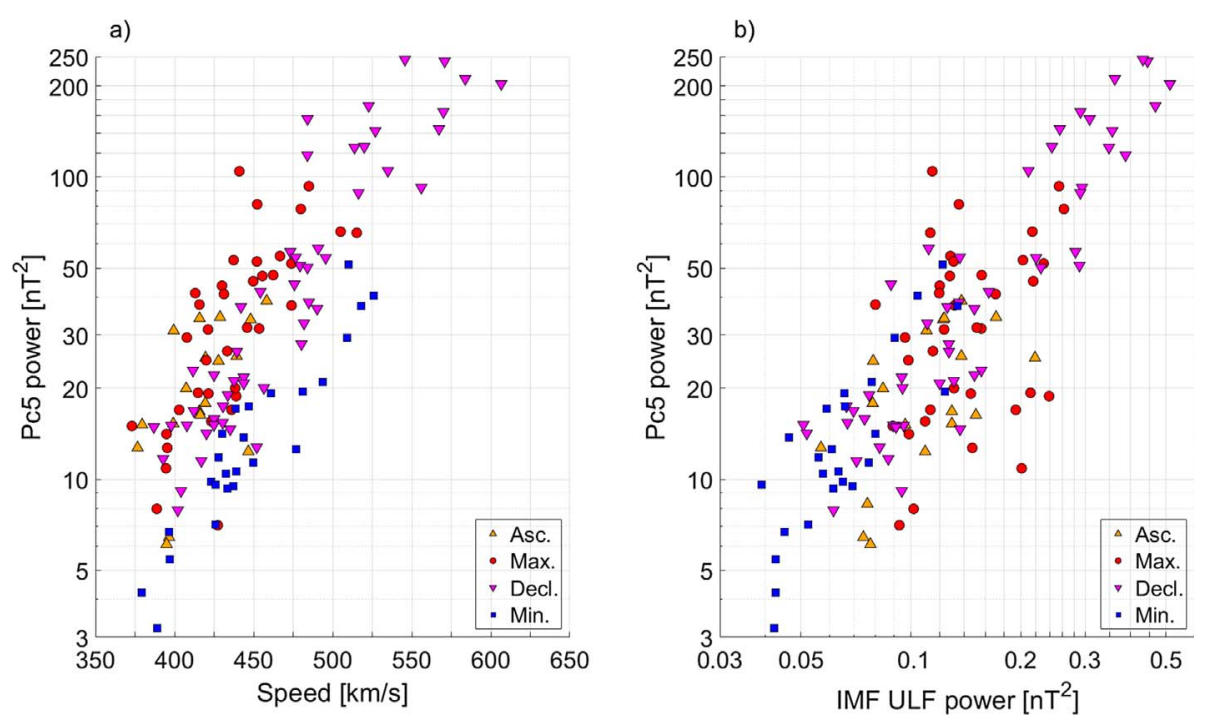

Fig. 2. Scatter plot showing $U_{\mathrm{KEV}}$ in comparison to a) radial solar wind speed, b) $U_{\mathrm{IMF}}$, color-coded by the solar cycle phase.

to occur at quite different times on ground and in solar wind. The largest ground ULF power in solar maximum is found in August 2000 with $104 \mathrm{nT}^{2}$. At the same time $U_{\mathrm{IMF}}$ is low compared to the peaks at the solar maximum, $0.11 \mathrm{nT}^{2}$. Respectively, the largest ULF power in IMF during solar maximum is in September 1999 when $U_{\text {IMF }}$ reaches $0.26 \mathrm{nT}^{2}$, coinciding with the ground ULF power is $78 \mathrm{nT}^{2}$ that raises to $93 \mathrm{nT}^{2}$ by October 1999. However, the second highest peak of $U_{\mathrm{IMF}}$ is in March 2002 corresponding to dip in ground ULF power that is as low as $19 \mathrm{nT}^{2}$. Despite the ambivalence of the maximum phase in general, the IMF and ground ULF power match better with each other during the early maximum phase, as seen e.g. around October 1999. While this agreement is not generally seen in the ascending phase, both the ULF power on ground and in solar wind fall sharply from April 1999 (flooring $6 \mathrm{nT}^{2}$ on ground, $0.07 \mathrm{nT}^{2}$ in solar wind) and they recover in August 1999 to culminate into the earlier described peak in October 1999.

To quantify the relation between the ground and ULF power in IMF, we produced scatter plots of ULF power at KEV against the solar wind speed and ULF power in IMF separately, shown in Figure 2. We then computed the linear (Pearson) correlation coefficient for $U_{\mathrm{KEV}}$ and $U_{\mathrm{IMF}}$, defined $r_{B B}=$ $r\left(\log U_{\mathrm{IMF}}, \log U_{\mathrm{KEV}}\right)$, and for $U_{\mathrm{KEV}}$ and radial solar wind speed $v_{r}$, defined $r_{v B}=r\left(v_{r}, \log U_{\mathrm{KEV}}\right)$. Correlation coefficients for different solar cycle phases are given in Table 2.

The 12 largest values for the ground Pc5 power are seen during the declining solar cycle phase while the three lowest values observed are seen during the solar minimum. The largest Pc5 is seen during very fast solar wind (monthly average $>530 \mathrm{~km} / \mathrm{s}$ ) and for large IMF fluctuations (monthly average $>0.28 \mathrm{nT}^{2}$ ). Ground Pc5 power can be predicted equally well based on solar wind speed $\left(r_{v B}=0.92\right)$ and IMF power $\left(r_{B B}=0.91\right)$. Pc5 power cannot be predicted very well during ascending phase by using either of the parameters nor any other tested parameters (such as particle density, not shown). If some parameter needs to be used during the ascending phase, this study proposes IMF power to be marginally better than solar wind speed. During solar maximum the solar
Table 2. Correlation coefficients between ground pulsation power and solar wind magnetic $\left(r_{\mathrm{BB}}\right)$ and solar wind speed fluctuations $\left(r_{\mathrm{vB}}\right)$.

\begin{tabular}{lcccc}
\hline Time interval & $r_{\mathrm{BB}}$ & $p$-value & $r_{\mathrm{vB}}$ & $p$-value \\
\hline Whole interval & .83 & $<0.001$ & .80 & $<0.001$ \\
Ascending phase & .59 & .007 & .56 & .016 \\
Solar maximum & .31 & .059 & .73 & $<0.001$ \\
Declining phase & .91 & $<0.001$ & .92 & $<0.001$ \\
Solar minimum & .84 & $<0.001$ & .93 & $<0.001$ \\
Decl. + minimum & .93 & $<0.001$ & .89 & $<0.001$ \\
\hline
\end{tabular}

wind speed works better than IMF power for predicting the ground Pc5 power.

Figure $2 \mathrm{a}$ shows that solar wind speed sets out a threshold for the ground Pc5. Ground power of $20 \mathrm{nT}^{2}$ cannot be exceeded during the solar minimum if the monthly solar wind speed stays below $450 \mathrm{~km} / \mathrm{s}$ and power of $30 \mathrm{nT}^{2}$ can be achieved when speed is larger than $500 \mathrm{~km} / \mathrm{s}$. In contrast, ground power reaches $100 \mathrm{nT}^{2}$ for $v=450 \mathrm{~km} / \mathrm{s}$ during the solar maximum and up to $160 \mathrm{nT}^{2}$ for $v=500 \mathrm{~km} / \mathrm{s}$ during the solar cycle declining phase. Minimum threshold has a roughly linear$\log$ relationship between solar wind speed and ground Pc5 power. We estimate that for the ground ULF power at KEV the following relationship holds:

$$
\min \left(U_{\mathrm{KEV}}\right) \approx 10^{0.0083 v_{r}-2.4} \mathrm{nT}^{2}, \quad \text { in units }\left[v_{r}\right]=\mathrm{km} / \mathrm{s} .
$$

This indicates that every $120 \mathrm{~km} / \mathrm{s}$ increase of mean solar wind speed multiplies the average ground ULF power by 10 . A similar threshold might be possible to find for $U_{\mathrm{IMF}}$ and $U_{\mathrm{KEV}}$ probably most easily for declining and minimum phases. Romanova \& Pilipenko (2009) have computed similar characterizations using a global ULF index derived by Pilipenko et al. (2008). The results presented in their paper match well with our results.

The monthly means of the logarithmic ULF powers (in 1998-2008) of interplanetary magnetic field $U_{\text {IMF }}$ (top-left) 
a)

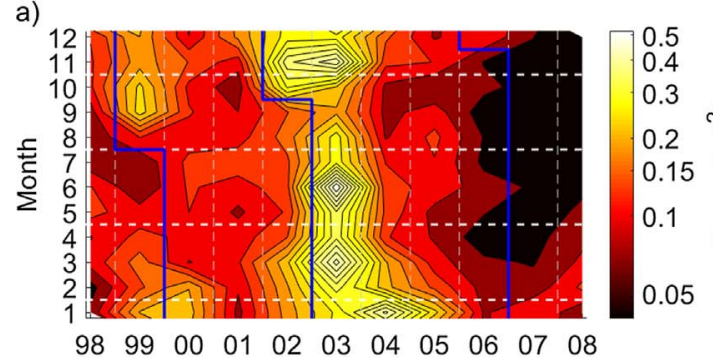

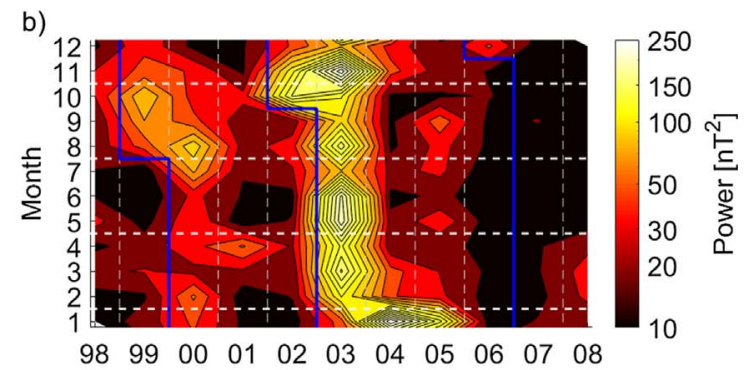

c)

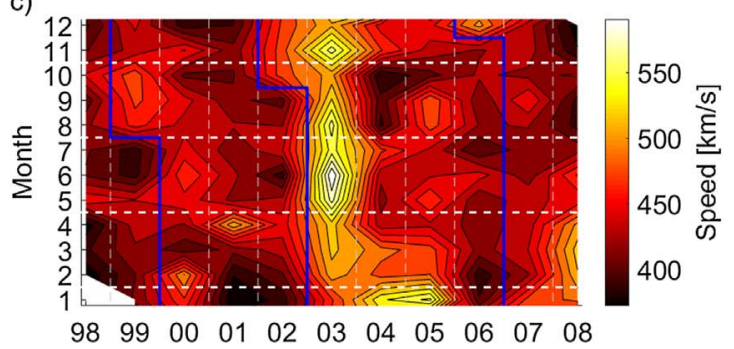

Fig. 3. Monthly mean value of (a) IMF ULF power, (b) ground ULF power, and (c) solar wind speed in 1998-2008. Each vertical bin contains the mean log monthly ULF power in (a) and (b), and the mean radial solar wind speed in (c). White dashed lines separate the figures by seasons, and blue solid lines show the places where the solar cycle phase changes.

and ground magnetic field in Kevo $U_{\mathrm{KEV}}$ (top-right) are shown in Figure 3, together with the monthly mean solar wind speed (bottom panel). At the top (bottom) of each figure the contours include the data of the next (last) year as well. The horizontal solid lines mark the boundaries of seasons, defined by months nearest to solstices and equinoxes: spring is Feb-Apr, summer is May-Jul, fall is Aug-Oct and winter Nov-Jan.

Considering the maximum monthly mean for each year, winter is the peak time of $U_{\mathrm{IMF}}$ (the upper panel of Fig. 3) for most years over the solar cycle 23 . In fact, the only years without a winter peak in $U_{\text {IMF }}$ were 1999 (fall equinox peak), 2003 (summer solstice peak in addition to winter and spring peaks), and 2008 (spring equinox peak). Peaks in the fall 1999, summer 2003 and fall 2003 are possibly due to the combination of coronal mass ejections and long-duration high-speed streams and related high fluxes of $\mathrm{MeV}$ electrons: their associated Pc5 activity produced high fluxes of $\mathrm{MeV}$ electrons in the magnetosphere (Borovsky \& Denton, 2006).

The solar wind speed has its peak month during equinoxes before year 2003, with the exception of 1998 when the peak speed is reached in summer. In 2003 the peak is in summer and throughout 2004-2007 in winter, with a spring equinox in 2008. $U_{\text {IMF }}$ and solar wind speed match each other in this respect during the declining phase of SC23.

On the ground there is a tendency for the yearly ground peak activity $U_{\mathrm{KEV}}$ to occur during equinoxes before year 2003: only year 1998 has the peak in summer solstice instead. After 2003, the yearly peak activity of $U_{\mathrm{KEV}}$ shifts to winter: all years 2003-2007 are winter-dominated. Only 2008 has its peak at spring equinox matching nicely to the seasonal pattern of $U_{\text {IMF }}$ in the same year. If the months immediately before and after a year are also contributed (December of the previous year and January of the following), the only difference is that the peak of 1998 in $U_{\text {IMF }}$ is switched from summer to winter solstice.
Considering the month of maximum ULF power for each year, we note that the month of peak activity on ground coincides with the peak solar wind speed in 9 out of 11 years: only years not coinciding are 2000 and 2003. The peak ULF activity in IMF coincides with peak solar wind speed in 6 years: 1998, and all of 2003-2007, all except 1998 winter-dominated years of ground ULF activity.

\section{Solar cycle evolution of latitudinal variability}

The ground ULF power was computed for all 30 IMAGE stations available for the time span 1998-2008 by the method described in Section 2. IMAGE stations are located in a longitudinal chain around $105^{\circ}$ magnetic longitude, corresponding to magnetic local time of MLT $=\mathrm{UT}+3 \mathrm{hr}$. For each station the data was further separated by the solar cycle phase (solar maximum: 1999.6-2002.7, declining phase: 2002.7-2006.9, solar minimum 2006.9-2009.0). The mean of the logarithmic power in each cycle phase was computed and shown separately for each station in Figure 4.

The mean ULF power at all stations is greater in the declining phase than at maximum or minimum. Independent of the solar phase, the ULF power raises with magnetic latitude until around $73^{\circ}$ Mlat. Solar maximum has clearly greater ULF power than the minimum up to $68^{\circ}$ but above $70^{\circ}$ the magnitude is similar in both phases. Only at $\operatorname{HOP}\left(73.06^{\circ}\right)$ the power at solar minimum is way lower than during other phases. This is likely because there is very little data available at HOP for the solar minimum.

Magnetic latitudes over about $71.6^{\circ}$ have L-shells computationally above 10 , which places them farther than the average distance of the magnetopause. Therefore their magnetic field 


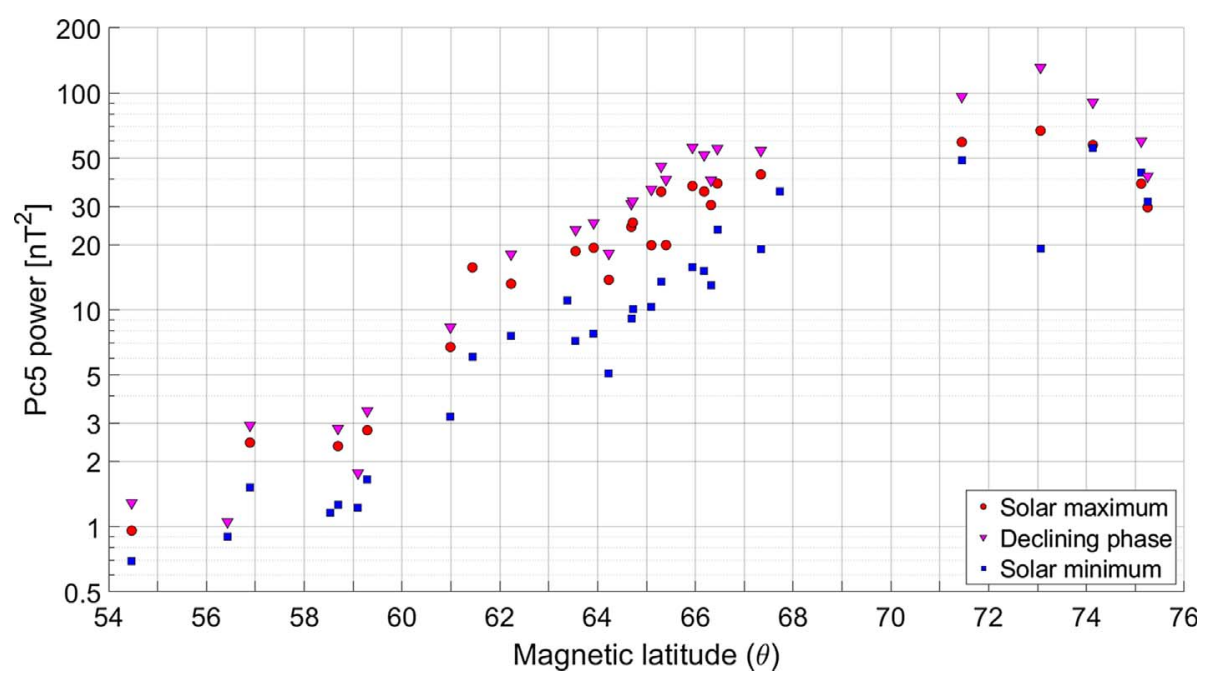

Fig. 4. Mean ULF power in all IMAGE stations for solar maximum (red), declining phase (purple) and solar minimum (blue).

lines map either into the solar wind or into Earth's magnetotail, depending on MLT. Hence, the ULF power for each station was further separated by MLT to day (MLT 09-15) and night (MLT 21-03) sectors. The result is shown in Figure 5.

The location of the largest ULF power depends on the MLT. For day-time, the largest power $\left(326 \mathrm{nT}^{2}\right)$ is recorded in Svalbard, at HOR station, $74.13^{\circ}$ magn. lat. In night-time the largest power $\left(156 \mathrm{nT}^{2}\right)$ in the data set is located in Norway's coast, at NOR station, $67.73^{\circ}$ magn. lat.

The night-time power is locally enhanced and larger than day-time power at the magn. latitudes $63^{\circ}-68^{\circ}$, roughly corresponding to auroral oval region, while elsewhere the day-time power is larger than night-time power. In this region, the nightside ground Pc5 power is on average roughly 2 times larger than the dayside Pc5 power. At magn. latitudes $71^{\circ}-75^{\circ}$ day-time ULF power exceeds the night-time ULF power.

The ULF power increases most rapidly between $58^{\circ}$ and $68^{\circ}$ magn. lat. when the increase is close to exponential with latitude. Within 9 degrees the ULF power increases roughly one and half orders of magnitude in both day-time and night-time power. One order of magnitude increase is observed between TAR $\left(58^{\circ}\right)$ and LYC $\left(61^{\circ}\right.$ magn. lat.), within 3 degrees. The typical mean ULF power in the southern Finland is of the order of 3-7 $\mathrm{nT}^{2}$, while in the northern Finland it is roughly 50-100 $\mathrm{nT}^{2}$. During day the largest ULF power is found at polar latitudes (at $72^{\circ}-74^{\circ}$ magn. lat.), while during the night they are found in the auroral oval, whose processes are controlled by the magnetotail processes. In the polar stations, at higher than $74^{\circ}$ magn. lat., the ULF power decreases rapidly regardless of MLT. These northernmost stations reside part of the time inside the auroral oval and otherwise within the polar cap depending on MLT and geomagnetic conditions (Xiong et al., 2014). The power decreases by a multiplier of 2-3 from the ULF power maximum towards the northernmost station (NAL) in the data set.

The different maximum location of ULF wave power implies that the day-time and night-time power are driven by different physical mechanisms: the night-time predominantly driven by magnetotail processes, and day-time by solar wind driven fluctuations. To estimate the relative importance of either

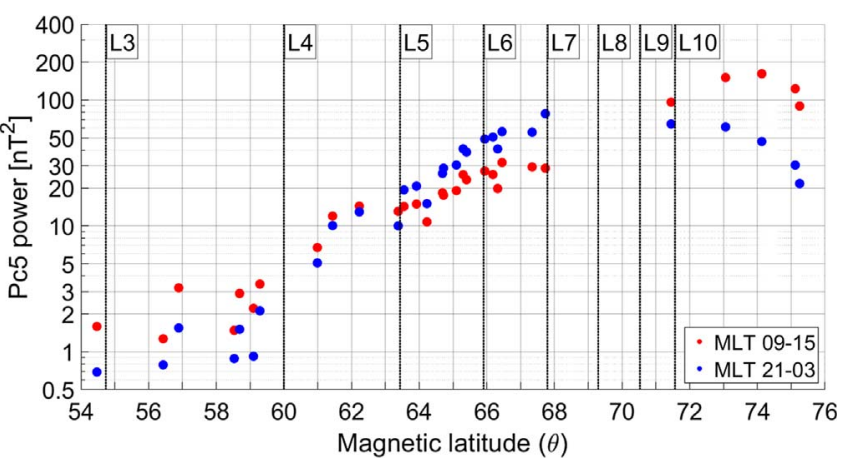

Fig. 5. Mean ULF power over years 1998-2008 for each IMAGE station separately for day (09-15 MLT) and night (21-03 MLT). The latitude of the respective $L$-shells (L3-L10) are shown by vertical lines.

mechanism, it would be required to study the location of the latitudinal power maximum in relation to changing solar wind and magnetospheric driving parameters. During the feasibility study for this paper we computed correlations between ground ULF wave power and different solar wind variables including solar wind speed, magnetic field, particle density, electric field and dynamic pressure. We found out the best correlation for IMF fluctuations ( $r=0.83-0.86$ depending on the vector component), the second best for radial solar wind speed $(r=0.80)$, and much lower correlations for all other parameters: particle density $(r=0.07)$, dynamic pressure $(r=0.71)$ and reconnection electric field $(r=0.12)$. That made us to decide to focus on the role of IMF wave power and leave the studies of other variables out of this paper.

\section{Discussion}

ULF waves are detected in many regions of the coupled Sun-Earth system. This paper studies ULF power in solar wind and on ground over monthly and solar cycle time scales. 
Ground ULF waves in Pc5 frequency range are known to be driven predominantly by solar wind speed (Tanskanen et al., 2005) and to be related to the Kelvin-Helmholtz mechanism (Nykyri, 2013) and auroral substorm processes (Pilipenko et al., 2001; Kozyreva et al., 2016). ULF waves on ground as well as in solar wind have been examined for decades (Jacobs et al., 1964). Stephenson \& Walker (2002) suggested, based on an event in April 1997, that Pc5 field line resonances may be directly driven by the solar wind oscillations. In their study, they found oscillations in discrete frequency bands within the solar wind, in the radial speed, thermal pressure, particle density and each component of the magnetic field. At the same time they found peaks in radar observations at similar frequencies. Similarly, Kepko et al. (2002) observed pressure and $B_{Z}$ fluctuations in the solar wind right before observing $B_{Z}$ magnetic fluctuations within the magnetosphere, implicating a driving relationship between compressional waves without and within the magnetosphere.

The Pc5 pulsations have been examined statistically e.g. by Mathie \& Mann (2001) and Takahashi et al. (2012). Mathie \& Mann (2001) studied the relationship of solar wind speed to daily ULF pulsation power for 1995, 1996 and 1997, an interval they corresponded to the declining phase of SC22. They found a strong positive correlation between solar wind speed and dayside magnetospheric pulsation power across the entire $L$-shell range they explored (3.75-6.79). Our results show that the correlation was also high for the entire declining phase of SC23 and it is true for stations at higher latitudes. Takahashi et al. (2012) dependence studied geomagnetic pulsations in a bit wider $L$-shell range 1.3-8.7 for years 2001 (solar maximum) and 2006 (solar declining phase) and compared the results to the solar wind data. They found (1) a positive correlation between the Pc5 amplitude and both solar wind speed and dynamic pressure, and (2) the amplitude at GOES and ground stations with $L>5$ to be higher in 2001 than in 2006 .

Our paper examines a long interval of data (1998-2008 covering SC23) compared to previous studies, as well as a wider range of magnetic latitudes $54.5^{\circ}-75.3^{\circ}$ (technically $L$-shells 3-15). Our finding that the highest ULF power was detected in the declining phase of SC23 matches nicely with the result of Mathie \& Mann (2001). Like Takahashi et al. (2012) we also find a strong positive correlation between magnetic fluctuations on the ground and solar wind speed but for monthly averages and for all years 1998-2008.

We find a very similar trend between solar wind speed and ground ULF power for the whole solar cycle, with the largest correlation coefficient in the solar minimum (0.93) closely followed by the declining phase (0.92) and lowest (0.56) in the ascending phase. In contrast, monthly ULF power in IMF and on ground correlate best in the declining phase (0.91), with a comparatively large value in the minimum (0.84) and worst in the solar maximum (0.31). However, the coefficient for both declining phase and solar minimum is 0.93 . Therefore, we conclude that IMF fluctuations may explain a part of the ground ULF power, especially during the declining phase of SC23, but less during the ascending phase and the solar maximum when solar wind speed and other quantities seem to dominate.

While investigating solar wind quantities, we determined the solar wind fluctuations in the proton density with the same method. The monthly mean of the density fluctuations was computed (not shown) and its correlation with the ground
ULF power at KEV was determined as 0.40 , greatly exceeded by the correlation 0.83 of the solar wind magnetic fluctuations. In the monthly time scales, the magnetic fluctuations appear more important than the density fluctuations for the ground ULF power.

The strong coupling between solar wind parameters and ground Pc5 pulsations over the declining phase is likely due to the large number of high-speed streams during this period (Xystouris et al., 2014). Earlier papers have reported Pc5 pulsation occurrence in the solar wind to correlate with spacecraft observations of magnetic field pulsations within the magnetosphere (e.g. Kepko et al., 2002; Kepko \& Spence, 2003) and on the ground (Regi et al., 2015), especially during high-speed streams (e.g. Kessel, 2008). High-speed streams are known to correlate with, and sometimes host, ULF fluctuations (see e.g. Engebretson et al., 1998; Kessel et al., 2004; Pahud et al., 2009; Potapov, 2013; Snekvik et al., 2013). During 2003 a particularly large number of high-speed streams were observed (e.g. Tanskanen et al., 2005; Xystouris et al., 2014). Combined with the observation that Pc5 pulsations are often associated with substorms it becomes likely that the high ULF power in both ground and solar wind measurements during the declining phase of the solar cycle are due to magnetic fluctuations of the interplanetary magnetic field, high-speed streams and the associated magnetospheric activity.

Early studies for solar cycles 18 and 19 have found Pc5 pulsations peaking in the minimum phase (Saito \& Matsushita, 1968; Rao \& Gupta, 1978). Our results for solar cycle 23 instead exhibit the maximum ULF power occurring in the declining phase of the cycle while the minimum phase is relatively weak. This might be explained by solar cycles 18,19 and 23 being fundamentally different from each other in this respect. In particular, the highly elongated coronal hole during the declining phase of solar cycle 23 (Mursula et al., 2016) caused high-speed streams to hit the Earth more regularly and over more extended time spans than during other solar cycles, and thus powered ULF waves. Similar elongated coronal holes are not reported to occur during the declining phases of the solar cycles 18 and 19.

The elongated coronal hole is also a candidate to explain some part of the seasonal peaks in the solar wind speed and by extension in the ground ULF power, because the associated high-speed solar wind defies regular seasonal patterns (Mursula et al., 2016) usually dictated by Russell-McPherron effect, equinoctial effect, and axial effect (Lockwood et al., 2016). The peak months of all three studied quantities in the declining phase of SC23 occur exclusively in the northern hemisphere winter, while the classic pattern of equinoctial peaks seems more typical in the preceding years. The peak months in both IMF power and ground ULF power closely resemble the ones reported for solar wind in Tanskanen et al. (2017).

The dependence of ground ULF wave power on the $L$-shell was studied by Mathie \& Mann (2001) who showed that ULF power increases towards higher magnetic latitudes. They reported an order of magnitude difference between the ULF powers in $L$-shells $L=4\left(60^{\circ}\right)$ and $L=6.6\left(67^{\circ}\right)$, which matches nicely with results presented here: we report an increase of about multiplier 20 over the same magn. lat. range. The $L$-shell range of $L=4$ to $L=8$ was studied by Rae et al. (2012) using data from CANOPUS/CARISMA network to include $L$-shells as high as $8\left(69.3^{\circ}\right)$ over years $1990-2005$. They found the ULF 
power in the dawn sector (03-09 MLT) to decrease around $L=8$. The same feature was seen in the spacecraft observations of the magnetosphere (Sarris \& Li, 2016) during a geomagnetic storm in October 2012. Our study expands the magnetic latitude range to $75.25^{\circ}$ geom. lat., corresponding to $L \approx 15$. Although we have no observations between $L=7\left(68^{\circ}\right)$ and $L=9\left(71^{\circ}\right)$, we found that the ULF power in the night sector (21-03 MLT) shows a trend consistent with Rae et al. (2012) results, with the maximum in correspondence to the nightside auroral oval, i.e. at $68^{\circ}$ or between $68^{\circ}$ and $71^{\circ}$ ); such power is likely due to processes related with substorms occurring in the magnetotail. On the other hand, the daytime (09-15 MLT) power peaks at $73^{\circ}-74^{\circ}$, at the poleward boundary of the dayside auroral oval. This peak is likely caused or at least largely influenced by near-noon pulsations, unrelated to Pc5 pulsations though occurring partially at the same frequencies (Pilipenko et al., 2018).

Ground ULF power depends on MLT (Fig. 5). In the auroral zone, roughly $63^{\circ}-68^{\circ}$, the night sector exhibits a larger ULF power than the day sector, but the opposite is true for both lower and higher latitudes. Our results show that the ULF power in the night sector begins to decrease at a way lower latitude (between $69^{\circ}$ and $71^{\circ}$ magn. lat.) than the power in the day sector (around $75^{\circ}$ magn. lat.). Lepidi \& Francia (2002) also studied ULF power over the years 1998-1999 (ascending phase of solar cycle 23) and over even greater latitudinal range, from magn. lat. $29^{\circ}$ (KAK station in Japan) to $85.5^{\circ}$ (THL station in Greenland) reporting local peaks between $70^{\circ}$ and $80^{\circ}$, with a sharper peak around $70^{\circ}$ during night-time and broader peak during day-time. Mostly, our results agree with those of Lepidi \& Francia (2002): the ULF power increases with latitude in a similar fashion. As IMAGE network does not have stations in the $68^{\circ}-73^{\circ}$ sector (except for BJN at $71.45^{\circ}$ ), in our results it looks like the power decreases in this interval. While our results agree with those of Lepidi \& Francia (2002), from our results the peak spot cannot be pin-pointed, although it exists in the auroral oval. Hence, the night sector power increase in the auroral zone is likely related to the substorm processes in the magnetotail or to the auroral electrojet. It is not clear, which mechanisms produce the Pc5 wave power in the auroral zone. However, based on studies on some features of storm-time substorms (Pilipenko et al., 2001; Kozyreva et al., 2016), Pc5 wave power variations in that region are connected to the auroral electrojet and fieldaligned currents in a way that is not presently understood. Higher than magn. lat. $70^{\circ}$ the day sector is dominating over night sector, in agreement with the study of magnetic fluctuations in Greenland by Peitso et al. (2018). This implies that most of the ULF power in this region is due to solar wind driven fluctuations. It is possible that some part of the ULF power in the high latitudes is generated by Kelvin-Helmholtz process at the magnetosheath/magnetosphere interface (Nykyri, 2013; Liou \& Sibeck, 2018), to which the magnetic latitudes therein are mapped by magnetic field lines. Magnetosheath being a complicated, turbulent region it is (Schwartz et al., 1996), further study is required to identify the dominant processes affecting the ULF power at $L>10$.

The lack of the magnetometers in the Norwegian Sea and Barents Sea regions, partly due to sparsity of ground suitable for magnetic observatories, is presently restricting the study of the magnetic activity in the high latitudes. This restriction could be lifted in the future by installing magnetometers in the seafloor. Alternatively, and more readily, this chain of magnetic latitudes could be studied by supplementing the data with measurements from other high-latitude magnetometers beside IMAGE network.

\section{Conclusions}

In this paper we have studied the monthly ULF power within solar wind and on ground over solar cycle 23 . The knowledge on spatial and time evolution of ULF power is useful for better understanding of how solar wind controls the internal magnetospheric fluctuations in different time-scales from daily and seasonal variability to solar cycle variability. The long-term and seasonal effects of the ULF waves need further investigation, as ULF waves have been suggested to modulate atmospheric parameters, at least at polar latitudes (Regi et al., 2016, 2017).

The main results of the paper are:

1. The highest ULF power in solar wind and on ground is observed in the declining phase of SC23, around 20022004, peaking in late 2003. The highest ULF power in IMF during the declining phase is about twice as high as the highest power in the solar maximum. Respectively, the ULF power on ground during the declining phase is three times the ground ULF power at maximum.

2. Ground ULF power $\left(U_{\mathrm{KEV}}\right)$ follows well the ULF power of the interplanetary magnetic field $Z$-component $\left(U_{\mathrm{IMF}}\right)$ and solar wind speed in the declining phase and the solar minimum. The correlation coefficient of $\log U_{\mathrm{KEV}}$ and $\log U_{\mathrm{IMF}}\left(v_{r}\right)$ is $r_{B B}=0.91\left(r_{v B}=0.92\right)$ for the declining phase and 0.84 (0.93) for the solar minimum. During ascending phase, both IMF power and solar wind speed are nearly equally effective $\left(r_{B B}=0.59\right.$ and $\left.r_{v B}=0.56\right)$, while during solar maximum the solar wind speed is a better predictor for ground ULF power $\left(r_{v B}=0.73\right)$ than the IMF power $\left(r_{B B}=0.31\right)$. The monthly solar wind speed sets a minimum for the ground ULF power roughly with a relation $\log \left(U_{\mathrm{KEV}}\right) \sim v_{r}$.

3. During ascending phase and solar maximum of solar cycle 23 the ULF power on ground has its peak season typically during an equinox, but after 2003 the maximum season changes from spring and fall to winter. The maximum yearly ULF power returns back to the equinoxes at the end of the time span examined here, i.e. in 2008. Thus, there is a shift from equinox-dominated years to solstice-dominated years in 2003.

4. The night-time ULF power exceeds the dayside power between $63^{\circ}$ and $68^{\circ}$ magn. lat., likely caused by substorms, matching with the typical auroral oval latitudes. The ULF power increases with magnetic latitude until certain location depending on MLT. The highest power in the night sector is reached at $68^{\circ}$ magn. lat. at Nordkapp (NOR), after which it decreases towards higher latitudes. The dayside power gives larger values than nightside power below $63^{\circ}$ (Dombås and Pello latitudes) and above $71^{\circ}$ magn. lat. Due to the data gap between $68^{\circ}$ and $71^{\circ}$ magn. lat. the exact latitude where nightside dominance changes to dayside dominance cannot be obtained from this data set. Furthermore, the latitude where 
the highest night-time power is reached cannot be concluded precisely due to the same reason. In the day sector the power increases until $74^{\circ}$ before decreasing towards higher latitudes. This implies that the ULF power in the highest latitudes is affected by solar wind-magnetosphere interactions such as the Kelvin-Helmholtz instability and interactions with IMF oscillations.

Acknowledgements. This work was supported by ReSoLVE Centre of Excellence of the Academy of Finland (projects 272157 and 307411) and COST Action ES-1005 (TOSCA) and G-EPOS infrastructure within EPOS and FIN-EPOS (number 293488 and 311714). We thank Goddard Space Flight Center and their CDAWeb service for providing the data from MAG and SWE instruments of ACE spacecraft. International sunspot number used in the determination of the phases of the sunspot cycle was acquired from WDC-SILSO, Royal Observatory of Belgium, Brussels. We would also like to thank Finnish Meteorological Institute and IMAGE network for providing their magnetic field data, and Prof. Tuija Pulkkinen from University of Michigan, Ann Arbor, and Dr. Andrew Dimmock from IRF Uppsala for useful discussions. The editor thanks Vyacheslav Pilipenko and an anonymous referee for their assistance in evaluating this paper.

\section{References}

Akasofu S-I. 1980. The solar wind-magnetosphere energy coupling and magnetospheric disturbances. Planet Space Sci 28(5): 495-509. https://doi.org/10.1016/0032-0633(80)90031-8.

Bentley S, Watt C, Owens M, Rae I. 2018. ULF wave activity in the magnetosphere: Resolving solar wind interdependencies to identify driving mechanisms. J Geophys Res Space Phys 123(4): 2745-2771. https://doi.org/10.1002/2017JA024740.

Borovsky JE, Denton MH. 2006. Differences between CME-driven storms and CIR-driven storms. J Geophys Res Space Phys 111 (A7): A07S08. https://doi.org/10.1029/2005JA011447.

Chiu MC, Von-Mehlem UI, Willey CE, Betenbaugh TM, Maynard JJ, et al. 1998. ACE spacecraft. Space Sci Rev 86(1): 257-284. https://doi.org/10.1023/A:1005002013459.

Clette F, Svalgaard L, Vaquero JM, Cliver EW. 2014. Revisiting the sunspot number. Space Sci Rev 186(1-4): 35-103. https://doi.org/ 10.1007/s11214-014-0074-2.

Clilverd M, Menk F, Milinevski G, Sandel B, Goldstein J, et al. 2003. In situ and ground-based intercalibration measurements of plasma density at $L=2.5$. J Geophys Res Space Phys 108(A10): 1365. https://doi.org/10.1029/2003JA009866.

Engebretson M, Glassmeier K-H, Stellmacher M, Hughes WJ, Lühr H. 1998. The dependence of high-latitude PcS wave power on solar wind velocity and on the phase of high-speed solar wind streams. J Geophys Res Space Phys (1978-2012) 103(A11): 26271-26283. https://doi.org/10.1029/97JA03143.

Fairfield DH, Cahill LJ Jr. 1966. Transition region magnetic field and polar magnetic disturbances. J Geophys Res 71(1): 155-169. https://doi.org/10.1029/JZ071i001p00155.

Frigo M, Johnson SG. 1998. FFTW: An adaptive software architecture for the FFT. In: Proceedings of the 1998 IEEE International Conference on Acoustics, Speech and Signal Processing, ICASSP'98 (Cat. No. 98CH36181), Vol. 3: 1381-1384. https://doi.org/10.1109/ICASSP.1998.681704.
Greene W. 1997. Econometric Analysis. Prentice-Hall international editions. Prentice Hall. ISBN 9780023466021 . https://books.google. fi/books?id=j09oTRcQ-xkC.

Hamming RW. 1973. Numerical Methods for Scientists and Engineers. Series in Pure and Applied Math. 2nd edn. Dover http://books.google.fi/books?id=Y3YSCmWBVwoC.

Harris FJ. 1978. On the use of windows for harmonic analysis with the discrete Fourier transform. Proceedings of the IEEE 66(1): 51-83. https://doi.org/10.1109/PROC.1978.10837.

Hynönen R. 2013. Geomagnetic activity and its sources during modern solar maximum. Master's thesis. University of Helsinki http://urn.fi/URN:NBN:fi-fe2017112252465.

Jacobs JA, Kato Y, Matsushita S, Troitskaya VA. 1964. Classification of geomagnetic micropulsations. J Geophys Res 69(1): 180-181 https://doi.org/10.1029/JZ069i001p00180.

Kangas J, Guglielmi A, Pokhotelov O. 1998. Morphology and physics of short-period magnetic pulsations. Space Sci Rev 83(3-4): 435-512. https://doi.org/10.1023/A:1005063911643.

Kepko L, Spence HE. 2003. Observations of discrete, global magnetospheric oscillations directly driven by solar wind density variations. J Geophys Res Space Phys 108(A6): 1-5. https://doi. org/10.1029/2002JA009676.

Kepko L, Spence HE, Singer H. 2002. ULF waves in the solar wind as direct drivers of magnetospheric pulsations. Geophys Res Lett 29(8): 39-1-39-4. https://doi.org/10.1029/2001GL014405.

Kessel R, Mann I, Fung S, Milling D, O'Connell N. 2004. Correlation of Pc5 wave power inside and outside themagnetosphere during high speed streams. Ann Geophys 22(2): 629-641. https://doi.org/10.5194/angeo-22-629-2004.

Kessel RL. 2008. Solar wind excitation of Pc5 fluctuations in the magnetosphere and on the ground. J Geophys Res Space Phys (1978-2012) 113(A4): 1-2. https://doi.org/10.1029/2007JA012255.

Kozyreva O, Pilipenko V, Engebretson M, Klimushkin DY, Mager P. 2016. Correspondence between the ULF wave power spatial distribution and auroral oval boundaries. Sol Terr Phys 2(2): 46-65. https://doi.org/10.12737/20999.

Lee L. 1982. Transmission of Alfvén waves through the rotational discontinuity at magnetopause. Planet Space Sci 30(11): 1127-1132. https://doi.org/10.1016/0032-0633(82)90123-4.

Lepidi S, Francia P. 2002. Low frequency (1-4 mHz) geomagnetic field fluctuation power: latitudinal dependence and relation with solar wind parameters. ESASP 477: 447-450.

Liou K, Sibeck DG. 2018. Dawn-dusk auroral oval oscillations associated with high-speed solar wind. J Geophys Res Space Phys 123: 600-610. https://doi.org/10.1002/2017JA024527.

Lockwood M, Owens MJ, Barnard LA, Bentley S, Scott CJ, Watt CE. 2016. On the origins and timescales of geoeffective IMF. Space Weather 14(6): 406-432. https://doi.org/10.1002/ 2016 SW001375.

Mathie R, Mann I. 2001. On the solar wind control of Pc5 ULF pulsation power at mid-latitudes: Implications for $\mathrm{MeV}$ electron acceleration in the outer radiation belt. J Geophys Res Space Phys 106(A12): 29783-29796. https://doi.org/10.1029/2001JA000002.

McComas D, Bame S, Barker P, Feldman W, Phillips J, Riley P, Griffee J. 1998. Solar wind electron proton alpha monitor (SWEPAM) for the Advanced Composition Explorer. The Advanced Composition Explorer Mission 563-612. https://doi.org/10.1007/ 978-94-011-4762-0_20.

Menk FW, Waters CL. 2013. Magnetoseismology: Ground-based Remote Sensing of Earth's Magnetosphere. John Wiley \& Sons, ISBN: 9783527652075.

Mursula K, Holappa L, Lukianova R. 2016. Seasonal solar wind speeds for the last 100 years: Unique coronal hole structures during 
the peak and demise of the Grand Modern Maximum. Geophys Res Lett 44, 30-36. https://doi.org/10.1002/2016GL071573.

Nykyri K. 2013. Impact of MHD shock physics on magnetosheath asymmetry and Kelvin-Helmholtz instability. J Geophys Res Space Phys 118(8): 5068-5081. https://doi.org/10.1002/jgra.50499.

Pahud D, Rae I, Mann I, Murphy K, Amalraj V. 2009. Ground-based Pc5 ULF wave power: Solar wind speed and MLT dependence. J Atmos Sol-Terr Phys 71(10): 1082-1092. https://doi.org/ 10.1016/j.jastp.2008.12.004.

Peitso P, Tanskanen E, Pulkkinen T, Mursula K. 2018. Highfrequency geomagnetic fluctuations at auroral oval and polar cap. Space Weather. https://doi.org/10.1029/2018SW001841.

Pilipenko V, Kozyreva O, Lorentzen D, Baddeley L. 2018. The correspondence between dayside long-period geomagnetic pulsations and the open-closed field line boundary. J Atmos Sol Terr Phys 170: 64-74. https://doi.org/10.1016/j.jastp.2018.02.012.

Pilipenko V, Romanova N, Simms L. 2008. ULF wave power index for space weather applications. In: EUR 23348 - COST Action 724 Developing the scientific basis for monitoring, modelling and predicting Space Weather, 230-237. Office for Official Publications of the European Union, Luxembourg, p. 230.

Pilipenko V, Watermann J, Popov V, Papitashvili V. 2001. Relationship between auroral electrojet and Pc5 ULF waves. J Atmos Sol Terr Phys 63(14): 1545-1557. https://doi.org/ 10.1016/S1364-6826(01)00031-1.

Potapov A. 2013. ULF wave activity in high-speed streams of the solar wind: Impact on the magnetosphere. J Geophys Res Space Phys 118(10): 6465-6477. https://doi.org/10.1002/2013JA019119.

Rae IJ, Mann IR, Murphy KR, Ozeke LG, Milling DK, Chan AA, Elkington SR, Honary F. 2012. Ground-based magnetometer determination of in situ Pc4-5 ULF electric field wave spectra as a function of solar wind speed. J Geophys Res Space Phys 117(A4): A04221. https://doi.org/10.1029/2011JA017335.

Rao DRK, Gupta JC. 1978. Some features of Pc5 pulsations during a solar cycle. Planet Space Sci 26(1): 1-20. https://doi.org/10.1016/ 0032-0633(78)90032-6.

Regi M, De Lauretis M, Francia P. 2015. Pc5 geomagnetic fluctuations in response to solar wind excitation and their relationship with relativistic electron fluxes in the outer radiation belt. Earth Planet Space 67(1): 9. https://doi.org/10.1186/s40623-015-0180-8.

Regi M, De Lauretis M, Redaelli G, Francia P. 2016. ULF geomagnetic and polar cap potential signatures in the temperature and zonal wind reanalysis data in Antarctica. J Geophys Res Space Phys 121(1): 286-295. https://doi.org/10.1002/2015JA022104.

Regi M, Redaelli G, Francia P, De Lauretis M. 2017. ULF geomagnetic activity effects on tropospheric temperature, specific humidity, and cloud cover in Antarctica, during 2003-2010. J Geophys Res Atmos 122(12): 6488-6501. https://doi.org/ 10.1002/2017JD027107.

Romanova N, Pilipenko V. 2009. ULF wave indices to characterize the solar wind-magnetosphere interaction and relativistic electron dynamics. Acta Geophys 57(1): 158-170.

Saito T, Matsushita S. 1968. Solar cycle effects on geomagnetic Pi 2 pulsations. J Geophys Res 73(1): 267-286. https://doi.org/ 10.1029/JA073i001p00267.

Sarris TE, Li X. 2016. Calculating ultra-low-frequency wave power of the compressional magnetic field vs. L and time: multispacecraft analysis using the Van Allen probes, THEMIS and GOES. Ann Geophys 34(6): 565-571. https://doi.org/10.5194/ angeo-34-565-2016.
Schwartz S, Burgess D, Moses J. 1996. Low-frequency waves in the Earth's magnetosheath: present status. Ann Geophys 14(11): 1134-1150. https://doi.org/10.1007/s00585-996-1134-z.

Simms L, Engebretson M, Posch J, Hughes W. 2006. Effects of the equatorward auroral boundary location and solar wind parameters on Pc5 activity at auroral zone stations: A multiple regression analysis. J Geophys Res Space Phys 111(A10): A10217. https://doi.org/10.1029/2005JA011587.

Simms L, Pilipenko V, Engebretson M. 2010. Determining the key drivers of magnetospheric Pc5 wave power. J Geophys Res Space Phys 115(A10): A10241. https://doi.org/10.1029/2009JA015025.

Smith CW, L'Heureux J, Ness NF, Acuña MH, Burlaga LF, Scheifele J. 1998. The ACE magnetic fields experiment. The Advanced Composition Explorer Mission 613-632. https://doi.org/ 10.1007/978-94-011-4762-0_21.

Snekvik K, Tanskanen E, Kilpua E. 2013. An automated identification method for Alfvénic streams and their geoeffectiveness. J Geophys Res Space Phys 118(10): 5986-5998. https://doi.org/ 10.1002 /jgra.50588.

Stephenson J, Walker A. 2002. HF radar observations of Pc5 ULF pulsations driven by the solar wind. Geophys Res Lett 29(9): 1297. https://doi.org/10.1029/2001GL014291.

Stewart B. 1861. On the great magnetic disturbance which extended from August 28 to September 7, 1859, as recorded by photography at the Kew Observatory. Philos Trans $R$ Soc London 151: 423-430. https://doi.org/10.1098/rstl.1861.0023.

Stone EC, Frandsen AM, Mewaldt RA, Christian ER, Margolies D, Ormes JF, Snow F. 1998. The advanced composition explorer. Space Sci Rev 86: 1-22. https://doi.org/10.1023/ A:1005082526237.

Takahashi K, Yumoto K, Claudepierre SG, Sanchez ER, Troshichev OA, Janzhura AS. 2012. Dependence of the amplitude of Pc5-band magnetic field variations on the solar wind and solar activity. J Geophys Res Space Phys 117(A4): https://doi.org/ 10.1029/2011JA017120.

Tanskanen E, Hynönen R, Mursula K. 2017. Seasonal Variation of High-Latitude Geomagnetic Activity in Individual Years. J Geophys Res Space Phys 122(10): https://doi.org/10.1002/2017JA024276.

Tanskanen E, Slavin J, Tanskanen A, Viljanen A, Pulkkinen T, Koskinen H, Pulkkinen A, Eastwood J. 2005. Magnetospheric substorms are strongly modulated by interplanetary high-speed streams. Geophys Res Lett 32(16): https://doi.org/10.1029/ 2005 GL023318.

Tanskanen EI. 2009. A comprehensive high-throughput analysis of substorms observed by IMAGE magnetometer network: Years 1993-2003 examined. J Geophys Res Space Phys (1978-2012) 114(A5): https://doi.org/10.1029/2008JA013682.

Xiong C, Lühr H, Wang H, Johnsen MG. 2014. Determining the boundaries of the auroral oval from CHAMP field-aligned current signatures-Part 1. Ann Geophys 32, 609-622. https://doi.org/ 10.5194/angeo-32-609-2014.

Xystouris G, Sigala E, Mavromichalaki H. 2014. A Complete Catalogue of High-Speed Solar Wind Streams during Solar Cycle 23. Sol Phys 289(3): 995-1012. https://doi.org/10.1007/s11207013-0355-z.

Yagova N, Lanzerotti L, Villante U, Pilipenko V, Lepidi S, Francia P, Papitashvili V, Rodger A. 2002. ULF Pc5-6 magnetic activity in the polar cap as observed along a geomagnetic meridian in Antarctica. J Geophys Res Space Phys 107(A8). https://doi.org/ 10.1029/2001JA900143.

Cite this article as: Hynönen R, Tanskanen EI \& Francia P 2020. Solar cycle evolution of ULF wave power in solar wind and on ground. J. Space Weather Space Clim. 10, 43. https://doi.org/10.1051/swsc/2020046. 